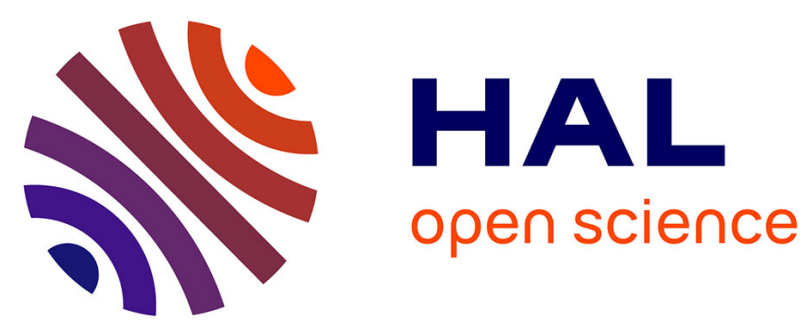

\title{
Anti-counterfeiting White Light Printed Image Multiplexing By Fast Nanosecond Laser Processing
}

Nicolas Dalloz, van Doan Le, Mathieu Hebert, Balint Eles, Manuel a Flores

Figueroa, Christophe Hubert, Hongfeng F Ma, Nipun Sharma, Francis

Vocanson, Stéphane Ayala, et al.

\section{To cite this version:}

Nicolas Dalloz, van Doan Le, Mathieu Hebert, Balint Eles, Manuel a Flores Figueroa, et al.. Anticounterfeiting White Light Printed Image Multiplexing By Fast Nanosecond Laser Processing. Advanced Materials, 2021, pp.2104054. 10.1002/adma.202104054 . ujm-03409454

\section{HAL Id: ujm-03409454}

\section{https://hal-ujm.archives-ouvertes.fr/ujm-03409454}

Submitted on 29 Oct 2021

HAL is a multi-disciplinary open access archive for the deposit and dissemination of scientific research documents, whether they are published or not. The documents may come from teaching and research institutions in France or abroad, or from public or private research centers.
L'archive ouverte pluridisciplinaire HAL, est destinée au dépôt et à la diffusion de documents scientifiques de niveau recherche, publiés ou non, émanant des établissements d'enseignement et de recherche français ou étrangers, des laboratoires publics ou privés. 


\title{
Anti-counterfeiting white light printed image multiplexing by fast nanosecond laser processing
}

\author{
Nicolas DALLOZ, Van Doan LE, Mathieu HEBERT, Balint ELES, Manuel A. FLORES \\ FIGUEROA, Christophe HUBERT, Hongfeng MA, Nipun SHARMA, Francis VOCANSON, \\ Stéphane AYALA, Nathalie DESTOUCHES*
}

N. Dalloz, V. D. Le, Dr. M. Hébert, B. Eles, M. A. Flores Figueroa, Dr. C. Hubert, Dr. H. Ma, Prof. F. Vocancon, Prof. N. Destouches

Univ Lyon, UJM-Saint-Etienne, CNRS, Institut d Optique Graduate School, Laboratoire Hubert Curien UMR 5516, 18 rue Professeur Benoît Lauras, Saint-Etienne, 42000, France E-mail: nathalie.destouches@univ-st-etienne.fr

\author{
N. Dalloz, Dr. N. Sharma \\ HID Global CID SAS, 33 rue de Verdun, Suresnes, 92100, France
}

S. Ayala

HID Global Switzerland SA, Z.I., Rte Pra-Charbon 27, Granges, FR 1614, Switzerland

\begin{abstract}
Passive plasmonic metasurfaces enable image multiplexing by displaying different images when altering the conditions of observation. Under white light, three-image multiplexing with polarization selective switching has been recently demonstrated using femtosecond-laser-processed random plasmonic metasurfaces. Here, the implementation of image multiplexing is extended, thanks to a color search algorithm, to various observation modes compatible with naked-eye observation under incoherent white light and to four-image multiplexing under polarized light. The laser-processed random plasmonic metasurfaces enabling image multiplexing exhibit self-organized patterns that can diffract light or induce dichroism through hybridization between the localized surface plasmon resonance of metallic nanoparticles and a lattice resonance. Improved spatial resolution makes the image quality compatible with commercial use in secured documents, as well as the processing time and cost thanks to the use of a nanosecond laser. This high speed and flexible laser process, based on energy efficient nanoparticle reshaping and self-organization, produces centimeter-scale customized tamper-proof images at low cost, which can serve as overt security features.
\end{abstract}

\section{Introduction}

Protecting the authenticity of banknotes, brands and identity documents is a major societal and economic concern. Most of the security features are based on optical effects with three levels of reading: overt (visible by eye, such as angle-dependent color-changing coatings), ${ }^{[1]}$ covert (requiring a revealing device such as a lens, a polarizer or a UV light source) ${ }^{[2-4]}$ or forensic (requiring heavy laboratory equipment). ${ }^{[5,6]}$ Overt security features are the first, and most of the time the only ones used to authenticate secure documents. However, forgery-proof overt security features are often based on wellknown technologies like holograms, ${ }^{[7]}$ microlens arrays ${ }^{[8]}$ or diffractive structures, ${ }^{[9]}$ which have a possibility of being counterfeited. Image multiplexing, which consists in encoding on a single metasurface the complete information of multiple images that can be displayed successively by switching the observation conditions, is a candidate for this security level provided that no revealing tool such as polarizer or optical lens are required. ${ }^{[10]}$ 
Image multiplexing has recently been enabled by the use of specific ink layouts ${ }^{[11,12]}$ and fabrication of plasmonic metasurfaces (metaldielectric nanostructured surfaces achieving unusual optical effects thanks to strong local field enhancements). The latter, have attracted much interest in the past decade for their expected technological impact in industry, with an improved manufacturability and recyclability of colored products. ${ }^{[13-15]}$ Structural colors that emerge from resonant interaction between light and these subwavelength nanostructured photonic layers are stable over time, exhibit high brightness, and can be controlled in different conditions of observation. So far, two types of image multiplexing based on plasmonic metasurfaces have been investigated in the literature. The first one is holographic image multiplexing, where different images are projected out of the metasurface plane through phase and amplitude manipulation. It demonstrates high storage capacity with an encoding which is based on wavelength, ${ }^{[16-20]}$ polarization, ${ }^{[20-25]}$ incidence angle, ${ }^{[26]}$ or angular momentum control $^{[27]}$ to switch between the multiplexed images. It also allows full color holographic patterns. ${ }^{[28,29]}$ The second one is printed image multiplexing, where the multiplexed images are displayed on the metasurface itself through the color control of each pixel in different observation modes and can be optimized in two ways. The first one is when the encoding is optimized for an observation under monochromatic light. Parameters such as wavelength, polarization and incidence angle are then used to switch between images and enable maximizing the number of multiplexed images. ${ }^{[30-34]}$ The second, when the metasurface is observed under incoherent white light, which implies much more constraints in terms of encoding. Here the number of multiplexed images is limited to two or three only, but this multiplexing strategy is the only one compatible with the production of overt security features observable with the naked eye in ambient light. However, all the outcomes published so far use polarizers to switch between the two or three multiplexed images, ${ }^{[35-38]}$ which prevents their use as overt security features.
Except our recent work, ${ }^{[37]}$ plasmonic metasurfaces used for image multiplexing involve electron or ion beam lithography. ${ }^{[35,36]}$ These technologies are difficult to transfer to an industry where the cost must be affordable and the fabrication processes are completely customized on a large scale. The customization of security features is indeed highly sought to prevent forgery, for instance including personal information of an ID card owner. ${ }^{[39]} \mathrm{At}$ present, laser processing appears to be the only technology that offers the flexibility to print customized large color plasmonic images, at low cost, with the ability to implement image multiplexing. Contrary to lithographic techniques, which remarkably control individual nanostructures, laser processing allows for tuning the statistical geometrical properties of nanoparticle assemblies, like their size-distribution, shape anisotropy or spatial distribution through self-organization mechanisms. ${ }^{[40-43]}$ Such a statistical control leads to what can be considered as random plasmonic metasurfaces, ${ }^{[44]}$ consisting of randomly distributed metallic nanostructures with certain size, density and orientation distributions, rather than well-ordered elements with an individually controlled size, position and orientation. This makes the metasurface harder to replicate, but also requires new ways of selecting the metasurfaces, as the link between the design parameters of the metasurface and its colors is difficult to model. Recently, the first implementation of printed three-image multiplexing has been demonstrated for white light observation using femtosecond laser processing of random plasmonic metasurfaces. ${ }^{[37]}$ However, these results require polarizers to observe the different images and involve expensive femtosecond lasers that are difficult to include in a profitable commercial solution.

Here, we demonstrate that a nanosecond laser (currently used in many industrial processes) can implement printed image multiplexing for high-end anti-counterfeiting applications (Figure 1) with several innovative assets, by leveraging the optical properties of these random plasmonic metasurfaces. This enables the use of non-polarized white light for the observation of overt security features. Image 
multiplexing is made possible by the singular features of the opto-geometrical properties of random plasmonic metasurfaces produced by such laser system, whose main features are unveiled in this work. Electromagnetic simulations are proposed to explain one of the mechanisms that are at the origin of the singular optical properties of such metasurfaces. Then, laser processing parameters for white light printed image multiplexing are found out among sets of experimental data by a specifically designed color search algorithm that can be applied to any technology producing mode-dependent colors. Two- and three-image multiplexing for naked eye observation (replaced by a camera for the article) under white light is demonstrated for different combinations of observation modes and various numbers of colors. Using polarized light, a four-image multiplexing is further demonstrated, using a blank image in the fourth mode. The image quality is optimized, by applying dithering printing two or more colors side by side to render the average color - on images, selecting the sets of metasurfaces providing the highest contrasts and improving the printing spatial resolution. The use of such nanosecond laser system also considerably decreases the processing time when compared to the previous works.

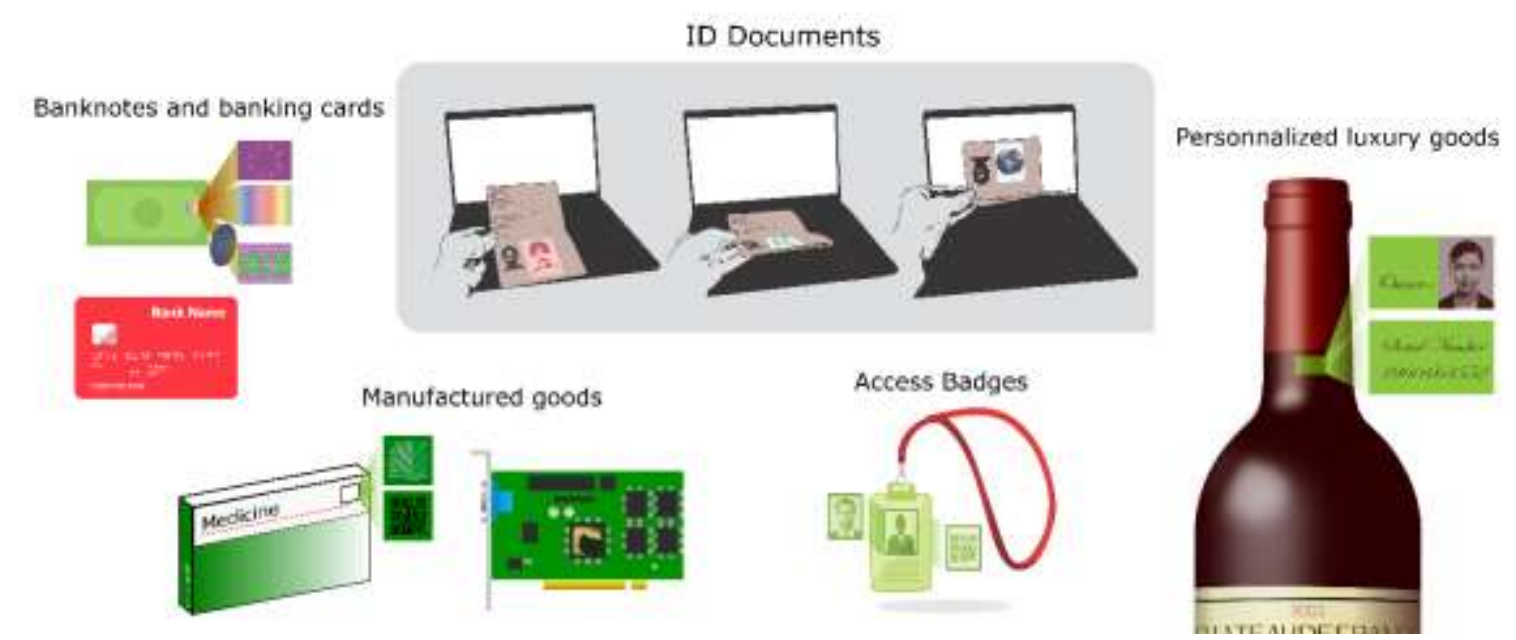

Figure 1. Applications of white light printed image multiplexing: secure and personalized anticounterfeiting protection for manufactured goods and documents.

\section{Results}

\subsection{Colors and observation modes}

A mesoporous film of $\mathrm{Ag}: \mathrm{TiO}_{2}$ (see Methods and Method S1, Supporting information, for details about the film elaboration) is processed with a focused nanosecond laser beam at 532 $\mathrm{nm}$ wavelength using various fluences, repetition rates, orientations of the linear laser polarization, scan speeds, and interline spacing or exposure time. Each singular set of laser processing parameters leads to the creation of a particular random plasmonic metasurface that is printed over a pixel whose size is chosen to be, in this article, either $72 \mu \mathrm{m}$ or $20 \mu \mathrm{m}$ (see Methods and Section 2 of the Supporting

Information for details about the nanosecond laser processing). A large amount (9 072) of metasurfaces made of $72^{2} \mu \mathrm{m}^{2}$ pixels (Figure
S3, Supporting information) have been produced by varying five laser parameters in order to create a database, from which the algorithm presented hereafter can find solutions to implement multiplexing. The same process was also carried out for $20^{2} \mu \mathrm{m}^{2}$ pixels to improve the resolution.

In the present section, we pay attention to a selection of 12 metasurfaces to present the interesting properties these random plasmonic metasurfaces exhibit. Figure 2 depicts the seven observation modes used in this article and show the colors (RGB values given in Table S2, Supporting information) and spectra (completed with Figure S5, Supporting Information) measured in each mode for the 12 metasurfaces under incoherent white light illumination. It also contains the color gamut in 
each mode, calculated from the 9072 printed metasurfaces. The convex hull plotted on each gamut (black dashed line in the gamuts) shows all colors accessible when implementing halftoning. The color of each metasurface depends on the observation mode. Five modes with non-polarized light are considered. They correspond to "front side reflection" (Figure 2a), "backside reflection" (Figure 2b), "scattering" (Figure 2c), "diffraction" (Figure 2d) and "transmission" (Figure 2e) configurations. In addition, two modes correspond to a configuration where the sample is observed in transmission between two polarizers crossed at $85^{\circ}$ from each other. These two modes, named "polarized transmission 1" (Figure 2f) and "polarized transmission 2" (Figure 2g) correspond to two azimuthal angles of the sample, $\alpha$ equals $45^{\circ}$ or $135^{\circ}$.

The widest color gamuts are obtained in "backside reflection", "scattering" and "polarized transmission $1 \& 2$ ", whereas the strongest lightness contrasts are obtained in "frontside reflection" and "unpolarized transmission" (e.g. A4 vs B1). Some metasurfaces, like $A 3$, are diffractive and light up in the "diffraction" mode, while other metasurfaces, like B3, which has the same color as A3 in the three transmission modes, do not diffract and remain black in the "diffraction" mode. Some metasurfaces also exhibit dichroic properties, i.e. a sensitivity of their complex refractive index to light polarization, as highlighted by squares $\mathrm{A} 6$ and B6, showing different colors in "polarized transmission 1" (green) and "polarized transmission 2" (purple) modes. Interestingly, while some metasurfaces are both diffractive and dichroic (B4), others are diffractive and non-dichroic (A3), or non-diffractive but dichroic (B6) or even neither diffractive nor dichroic (B1).

As better evidenced in the next section, dedicated to the nanoscale characterization of the laser-induced random plasmonic metasurfaces, the diffraction is due to the presence of laser-induced self-organized gratings. The low period ( $<400 \mathrm{~nm}$ ) of such gratings makes them highly dispersive for visible light, and hence explains why a single color (blue for the chosen angles in the "diffraction" mode) reaches the camera sensor. In Figure $2 \mathrm{~d}$, all the gratings present in squares $\mathrm{A} 3, \mathrm{~A} 4, \mathrm{~B} 4$, and $\mathrm{B} 5$ are perpendicular to the incidence plane, in which the detector is placed. When rotating the gratings in their plane, conical diffraction occurs, and a detector placed in the plane of incidence cannot catch diffraction anymore. A diffractive grating whose orientation is not perpendicular to the incidence plane can thus appear black in the diffraction mode. ${ }^{[45]}$ The grating orientation is then used as an encoding parameter for multiplexed diffractive images later in this article. As illustrated in the next section, the grating orientation depends only on the laser polarization orientation. 


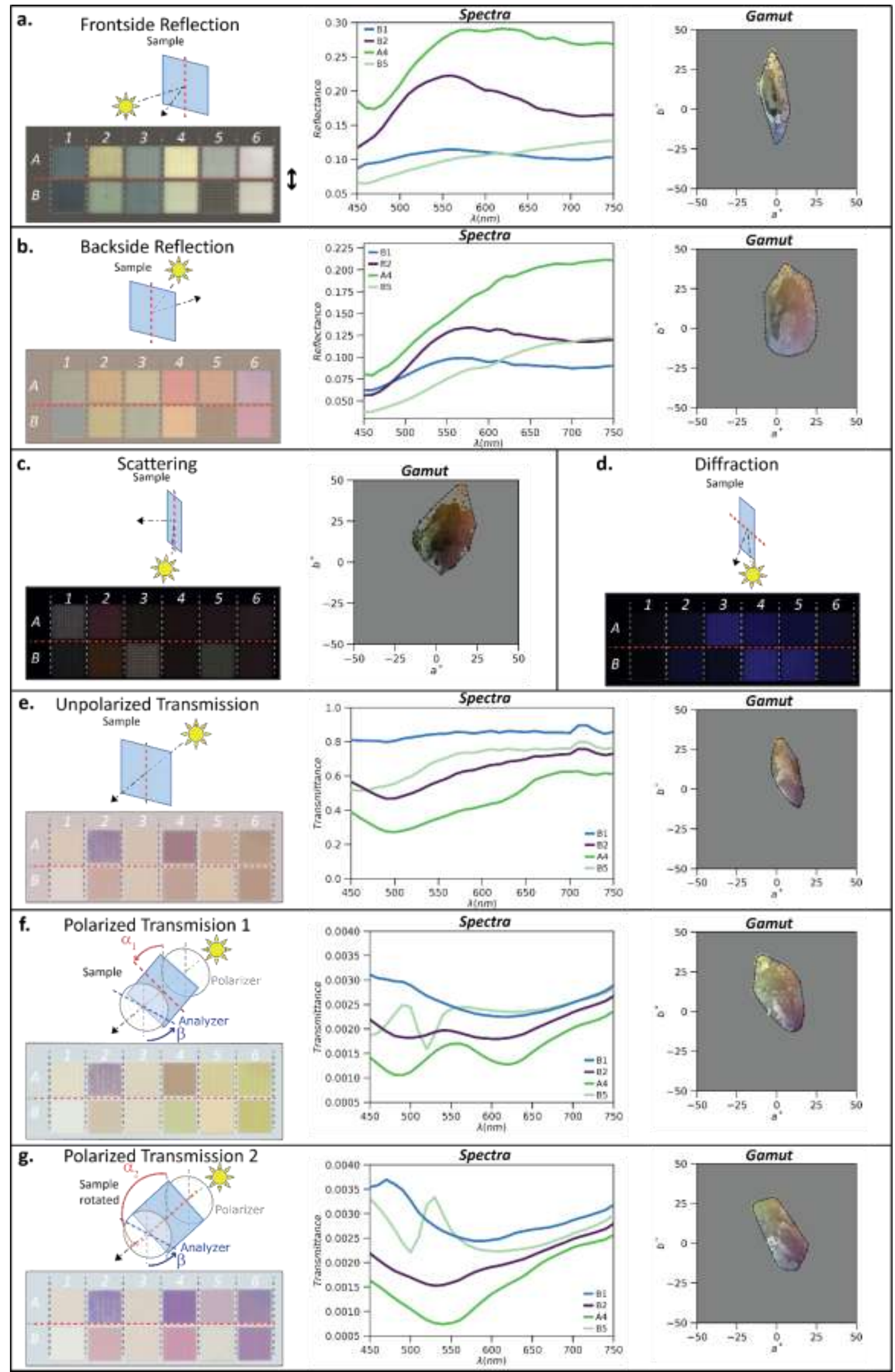


Figure 2. Colors of 12 laser-processed metasurfaces with a selection of 4 spectra (the other 8 spectra are in Figure S5, Supporting information), and overall color gamuts in CIE a*b* plane from 9072 metasurfaces, recorded in 7 observation modes. For each observation mode, the sketch illustrates the optical setup used to record the color images (setup described in Figure S7, Supporting information). All details about the measurements are given in the Methods section. The laser processing parameters used to print the 12 squares are given in Table S1, Supporting information. The laser polarization (double-sided black arrow shown in a) is parallel to the vertical side of all squares (laser polarization oriented at $0^{\circ}$ ). The dashed red line in all sketches represents the orientation of the horizontal side of squares. The background surrounding the color images shows the average color of the unprocessed film in the considered modes. Using the background color present during real observations gives the reader a better perception of colors. Indeed, setting this background to a white color in all modes would change the chromatic adaptation process and spatial effects such has simultaneous contrast would happen. Sensitivity limitations prevented the spectral measurements in the scattering mode. No gamut is shown in diffraction as color is angle-dependent and only the brightness variation is used in this mode. The side of each square is $0.864 \mathrm{~mm}$ long.

\subsection{Nanoscale properties of laser- induced plasmonic surfaces}

In last years, femtosecond laser processing of similar films was largely investigated, demonstrating the growth and reshaping of silver nanoparticles, their self-organization along embedded or surface gratings, as well as three dimensional self-organization. ${ }^{[40,41,45]}$ However, for industrial applications, femtosecond lasers appear to be very expensive, and this led us to consider processing by nanosecond lasers already largely used for personalized grey level marking in the security market. The results reported below are the first ones demonstrating nanosecond-laser-induced self-organization of silver nanoparticles in $\mathrm{TiO}_{2}$ thin films.

The nanosecond pulse duration (1.3 ns) strongly alters the mechanisms leading to the nanoparticle growth. As expected with higher thermal effects, the number of pulses required to grow silver nanoparticles and get various colors is smaller than what is required with a femtosecond laser at similar repetition rate. Compared to the $10 \mathrm{~mm} \mathrm{~s}^{-1}$ scan speed used to print multiplexed images with a femtosecond laser (190 fs pulse duration) at $515 \mathrm{~nm}$ wavelength and $500 \mathrm{kHz}$ repetition rate, ${ }^{[37]}$ the multiplexed images presented here involve scan speeds up to $2000 \mathrm{~mm} \mathrm{~s}^{-1}$. Thus, implementing nanosecond laser processing reduces the printing time of multiplexed images on centimeter scale in average by about a factor of 50 .

Despite the much higher processing speed, self-organization mechanisms also take place when using a nanosecond laser. Although this article cannot describe in detail the high variety of metasurfaces produced by nanosecond laser, a selection of characteristic selforganized structures exhibiting dichroism, diffraction or both simultaneously, is presented in Figure 3. This selection gives the reader an overview of the nanostructure properties at the origin of the singular colors required for multiplexing. At low fluence $(69 \mathrm{~mJ}$ $\mathrm{cm}^{-2}$ ), Ag nanoparticles tend to grow near the top surface and align roughly along the laser polarization, making the metasurface dichroic for both speeds, as highlighted by the different colors recorded in the two polarized modes. When comparing the 15 and $125 \mathrm{~mm} \mathrm{~s}^{-1}$ speeds, it appears that bigger nanoparticles are grown at lower speed even though a large density of small Ag nanoparticles still fills the metasurface. At the fluence of $151 \mathrm{~mJ} \mathrm{~cm}^{-2}$, self-organized gratings are formed on the film surface and the $\mathrm{Ag}$ nanoparticle size remains small in overall. A modulation of the nanoparticle density between the grating grooves and ridges characterizes the metasurface at high speed at this fluence. At $414 \mathrm{~mJ} \mathrm{~cm}^{-2}$, the grating grooves are deeper and the $\mathrm{Ag}$ nanoparticle density is lower. A stronger topographic contrast appears at low speed in this case. The period of the self- 
organized gratings lies in the range between $290 \mathrm{~nm}$ and $340 \mathrm{~nm}$. The grating lines are parallel to the incident laser polarization. Interestingly, the anisotropy of the nanogratings with the highest contrast at higher fluence is negligible, meaning that the optical anisotropy is more related to the presence of large nanoparticles or a high density of small nanoparticles preferentially aligned in one direction than to the presence of surface grating-like structures. Conversely, the diffraction efficiency is much higher for the surface grating-like structures obtained at high fluence than for metasurfaces with bigger nanoparticles obtained at low fluence. At intermediate fluences, the modulation of the nanoparticle density observed at high speed also diffracts light. As the period value is lower than the incident wavelengths (white light), the $-1^{\text {st }}$ diffracted order only appears for an incidence angle larger than $10^{\circ}$ for the blue $(400 \mathrm{~nm})$ part of the spectrum and is measured in the backscattering configuration described in Figure 2. When comparing these metasurfaces with the ones produced by femtosecond lasers, ${ }^{[37,40,41,45]}$ the embedded nanoparticles can have higher sizes at low speed and the surface grating-like structures are parallel to the laser polarization while they were perpendicular to the laser polarization with the femtosecond laser processing.

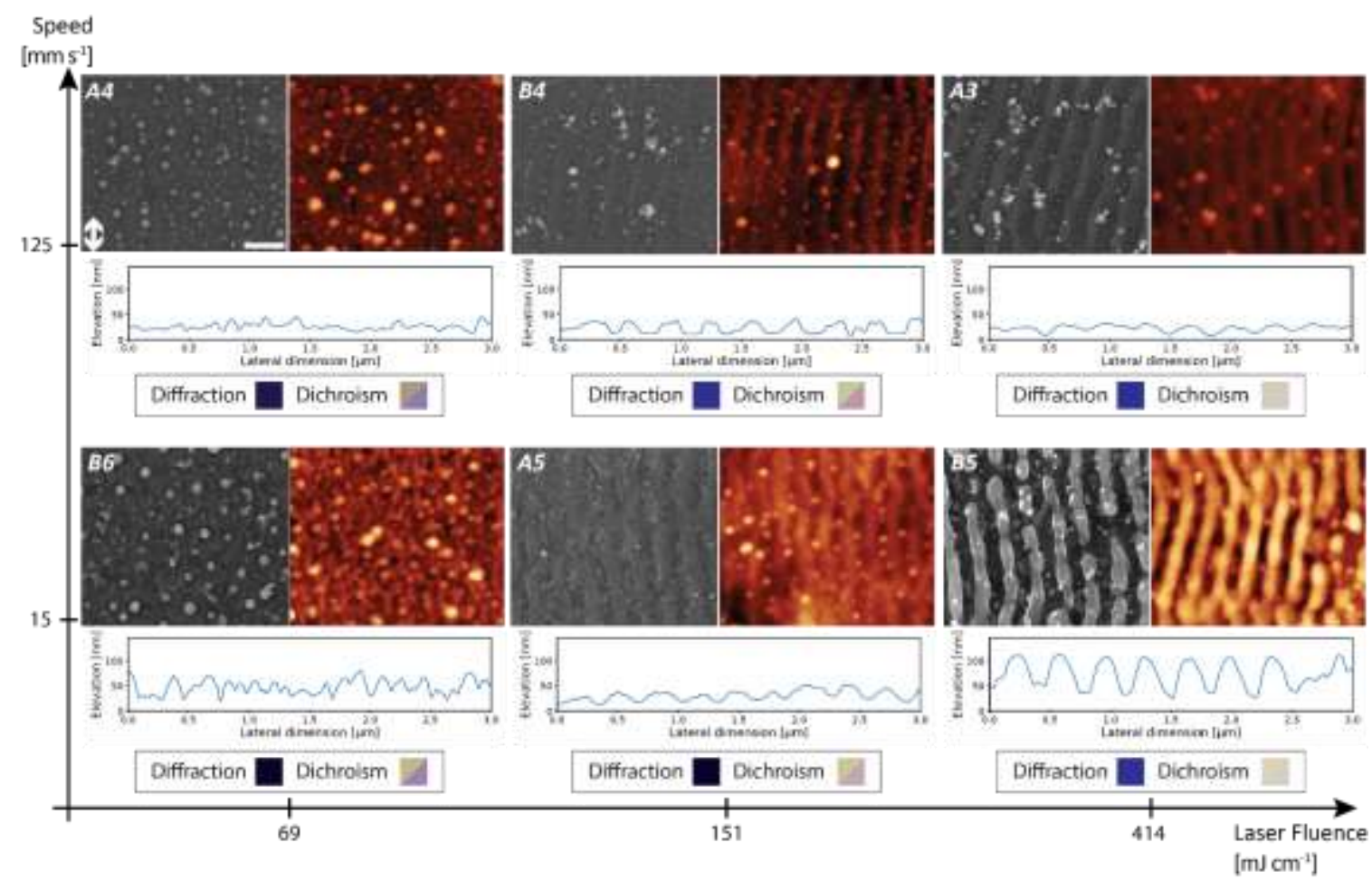

Figure 3. Nanostructured metasurfaces produced by ns laser processing. SEM and AFM micrographs and topographic profile perpendicular to laser polarization, of six of the twelve metasurfaces shown in Figure 2, obtained at various scan speeds and laser fluences. At this repetition rate $(300 \mathrm{kHz})$ and with the $1 / \mathrm{e}$ laser spot size of $13.5 \mu \mathrm{m}$, the speeds $15 \mathrm{~mm} \mathrm{~s}^{-1}$ and $125 \mathrm{~mm} \mathrm{~s}^{-1}$ correspond to an effective number of pulses of 1080 and 129.6 , respectively. The colors in "diffraction", and in "polarized transmission 1/2" highlighting the sample dichroism, are reminded for each structure. The scale bar is the same for all SEM and AFM images and corresponds to $500 \mathrm{~nm}$. The double-sided white arrow gives the direction of the laser polarization during processing. The laser scanning direction for printing the squares is at $45^{\circ}$ from the double arrow (see Supporting Note 1). 


\subsection{Origin of dichroism in self-organized patterns of nanoparticles}

The presence of metallic nanoparticles in the thin $\mathrm{TiO}_{2}$ film and subwavelength gratings is at the origin of various optical phenomena, such as localized surface plasmon resonances, thin film interferences, diffraction, and hybridization of resonances. In this section, we undertake electromagnetic simulations with a homemade code described in the Methods section to evidence the excitation of lattice modes in the self-organized patterns of nanoparticles, and explain one cause of dichroism.

The considered metasurface (scan speed: 15 $\mathrm{mm} \mathrm{s}^{-1}$; fluence: $75 \mathrm{~mJ} \mathrm{~cm}^{-1}$ ) is characterized by scanning electron microscopy (top view) and scanning transmission electron microscopy (cross-section) and shows a broad size distribution of $\mathrm{Ag}$ nanoparticles with two main peaks (Figure 4a-c). Such a double peak size distribution was also obtained when processing the same kind of samples with a $\mathrm{cw}$ visible laser, and it originates from coalescence processes between growing silver nanoparticles ${ }^{[46]}$. The larger nanoparticles are roughly aligned near the bottom side of the film along grating lines whose period is estimated to $297 \pm 35 \mathrm{~nm}$ and most of the nanoparticles are embedded within a thin film of $90+10 \mathrm{~nm}$ thickness. Energy dispersive X-ray spectra (not shown) demonstrates that the film is mainly composed of $\mathrm{Ti}$ and $\mathrm{O}$ and also contains $\mathrm{Si}$ atoms. This metasurface does not diffract light but exhibits strong dichroism, which is characterized by a polarization sensitive transmission. For TM polarization (electric field perpendicular to the grating lines and parallel to the $x$ axis), the transmission shows a broad dip that covers the whole visible range with a minimum around $468 \mathrm{~nm}$. For TE polarization, the transmission exhibits two dips at $468 \mathrm{~nm}$ and $658 \mathrm{~nm}$. To analyze these spectra, a simplified system has been considered for simulations. It is composed of $\mathrm{Ag}$ nanoparticles of diameter $\mathrm{D}$, embedded in a $90 \mathrm{~nm}$ thick film of refractive index $\mathrm{n}=2$, and perfectly aligned on the bottom side of the film with a period of $297 \mathrm{~nm}$. The substrate is glass of refractive index $n_{s}=1.5$, and the superstrate air. The simulations are carried out with a limited number of nanoparticles, 10, which broadens the delocalized resonances compared to an infinite grating. In a certain way, this allows to take into account the loss of coherence due to irregularities in the selforganized periodic pattern. Another numerical approach consists in introducing disorder in the nanoparticle pattern, but this requires involving a large number of nanoparticles to get good statistical results, which is heavy and time consuming.

Simulations carried out with nanoparticle diameters belonging to the experimental size distribution (Figure S6a) show that below 20 $\mathrm{nm}$, the extinction cross section is independent on polarization and exhibits a dipolar mode below $500 \mathrm{~nm}$. For diameters larger than 30 $\mathrm{nm}$, higher order modes progressively appear and all modes increase in amplitude and shift to the red for increasing diameter. The dipolar mode becomes very polarization dependent contrary to the higher order modes. To compare the simulations with the experiment, a weighted average of the extinction crosssection is calculated accounting for the size distribution of nanoparticles (Figure $4 \mathrm{~b}$ ) and is used to estimate the transmission coefficient of the film (see Method for details). While the simple averaging does not rigorously account for the true sample and may explain the overall higher transmission of the simulated results, the latter has features very similar to the transmission coefficient of the sample (Figure 4e). To better explain the origin of dichroism in this sample, we now focus on one particular diameter, whose spectrum is close to the average spectrum, $D=80 \mathrm{~nm}$ (Figure $4 \mathrm{f}$ ).

The extinction cross section in transmission shows that the dipolar resonance is shrunk and enhanced for the TE polarization compared with the TM one. This behavior can be attributed to the far-field coupling between scatterers through diffraction, which is much higher for TE than for TM polarization, and which leads to a so-called lattice mode ${ }^{[47,48]}$. The latter is well documented in the literature $^{[49]}$ and occurs near the Rayleigh anomaly, i.e. when a diffraction order is grazing on the surface. ${ }^{[48,50]}$ This is also referred as a Fano-type resonance where the broad dipolar mode of the localized surface plasmon 
resonance (LSPR) of silver nanoparticles spectrally overlaps the narrower propagating mode (delocalized resonance) and interferes to create hybrid states. The LSPR at the surface of the nanoparticles can then be attenuated or strengthened, as shown hereafter.

Near field mappings have been calculated at three characteristic wavelengths for TE (Figure 4g-i) and TM (Figure S6b) polarizations. They highlight the stronger near field at the nanoparticle surface for TE than for TM at 670 $\mathrm{nm}$ (dipolar mode) and a similar surface enhancement at $506 \mathrm{~nm}$ (quadrupolar mode). In the 550-600 $\mathrm{nm}$ range, the TE extinction cross-section is significantly attenuated compared with the TM one. The near field mappings at $546 \mathrm{~nm}$ highlight the excitation of a delocalized mode by the dipolar radiation (the near field of the dipolar mode only, has also been added (Figure 4k) to stress the role of the dipolar mode in the excitation) for TE that strongly attenuates the dipolar field on the nanoparticle surface (the quadrupolar mode is also present at this wavelength). This leads to a lower absorption than for TM polarization.

Further developments will be needed to better explain other interesting optical properties of these metasurfaces, such as the narrower gamut obtained in frontside reflection than in backside reflection. Such an insight into the electromagnetic modeling of the nanosecondlaser-induced metasurfaces clarifies the fact that predicting the color changes exhibited by all metasurfaces is still not achievable by physical models. We thus propose an alternative method to find out, among a large database of metasurfaces produced by varying the laser processing parameters, those that are interesting for application in multiplexing. 

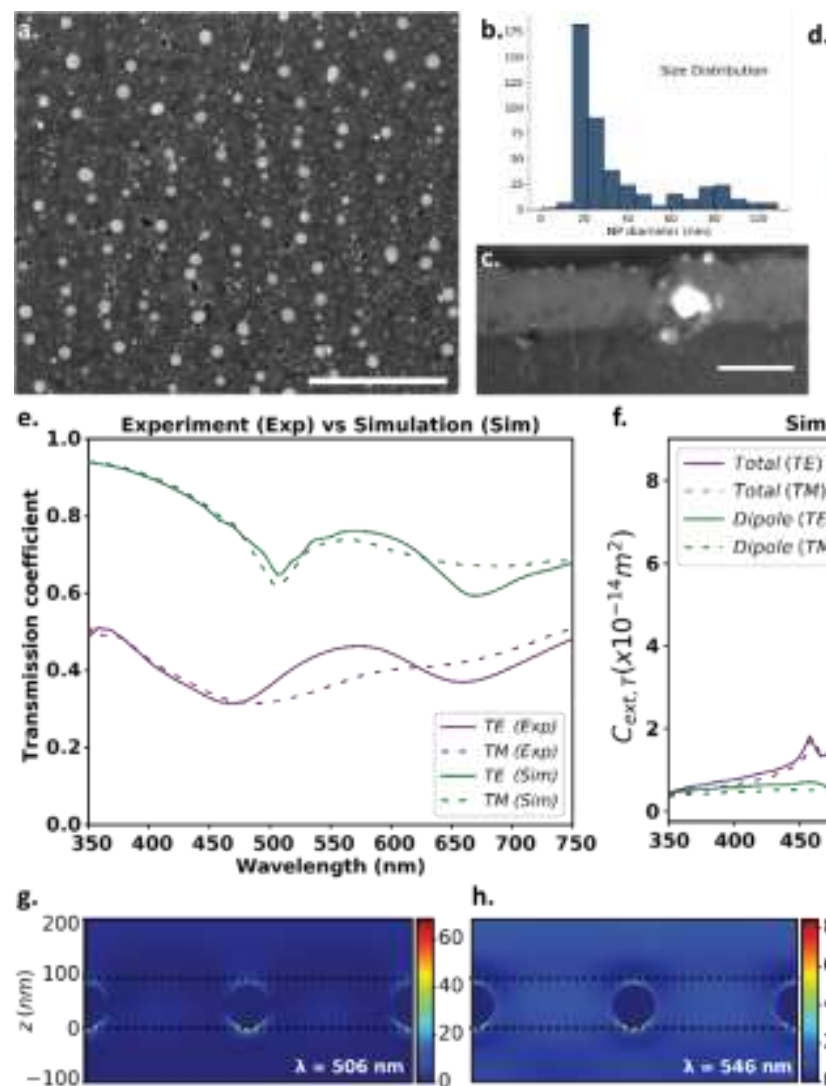

h.
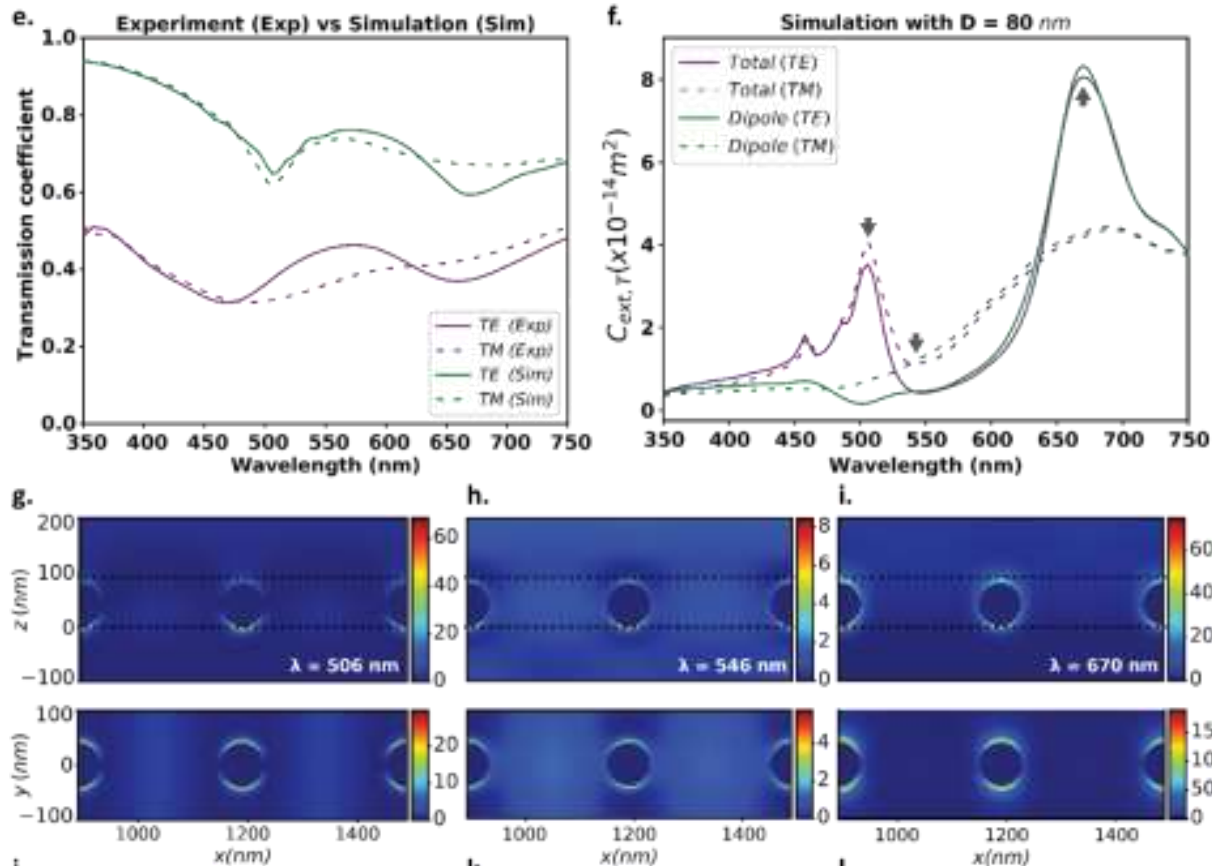

j.

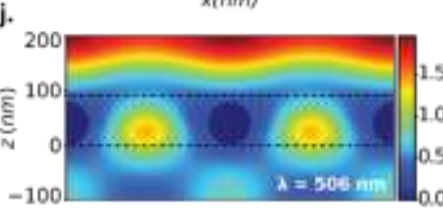

k.

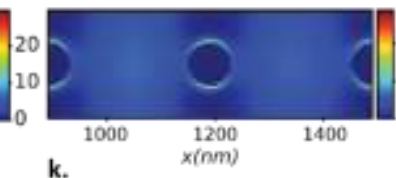

(n)
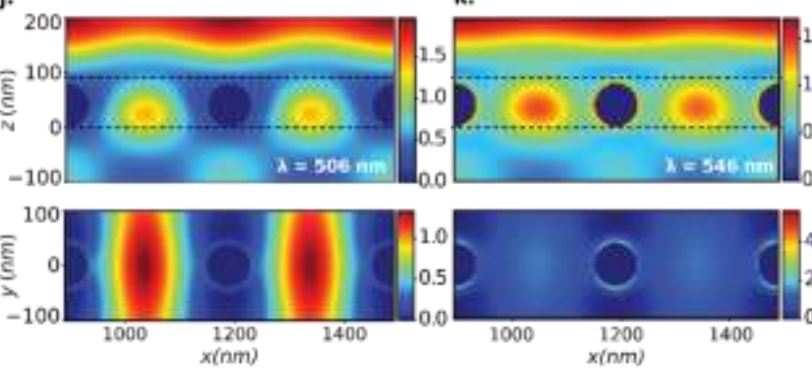

d.
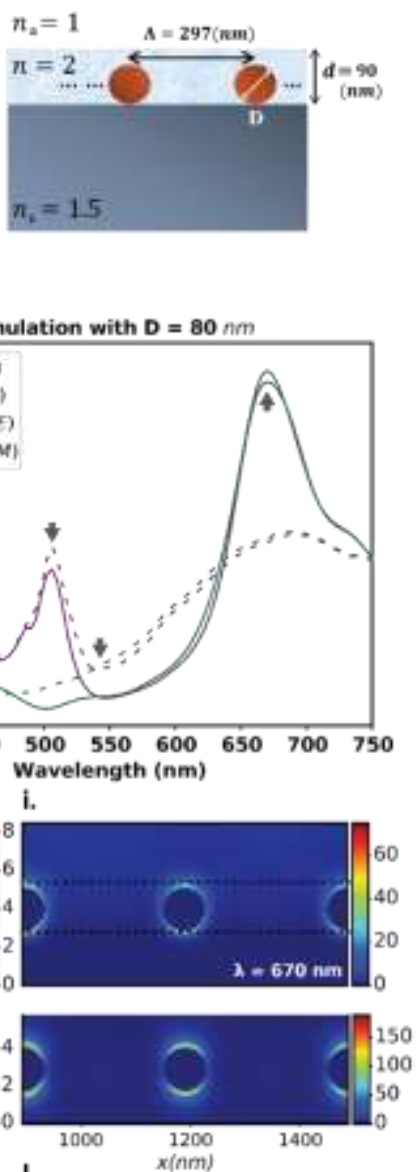

1.
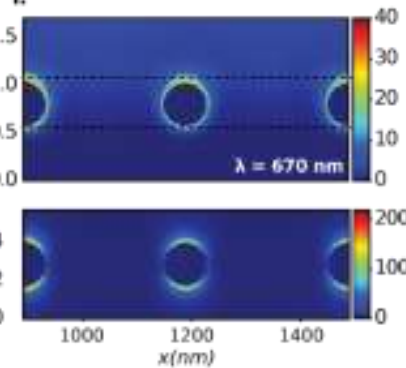

Figure 4. Electromagnetic simulations. (a) SEM micrograph of the considered metasurface. Scale bar is $1 \mu \mathrm{m}$. (b) Size distribution of the silver nanoparticles inferred from SEM images. (c) TEM cross section showing from top to bottom: the air, the $\mathrm{TiO}_{2}$ layer with one big nanoparticle, the glass substrate. Scale bar is $100 \mathrm{~nm}$. (d) Corresponding modeled structure consisting of 10 nanoparticles of diameter $\mathrm{D}$, separated by $297 \mathrm{~nm}$, embedded in a $90 \mathrm{~nm}$ thick $\mathrm{TiO}_{2}$ layer. (e) Transmission spectra measured on the sample and simulated from the weighted average of extinction cross section in transmission calculated for various diameters, for TE and TM polarizations. The nanoparticle diameter is varied from $10 \mathrm{~nm}$ to $90 \mathrm{~nm}$ by step of $10 \mathrm{~nm}$ (detailed calculations in Figure S6) (f) Simulated extinction cross section for D $=80 \mathrm{~nm}$, calculated with 3 harmonics (total) or with 1 harmonic corresponding to the dipolar mode (dipole). Arrows indicate the wavelengths selected for the near field images: 506, 546 and 670 $\mathrm{nm}$. (g-i) Cross sections and top views of the simulated near field (TE) with three harmonics at the selected wavelengths. For $(x, z)$ cross sections, $y=0 \mathrm{~nm}$. For $(x, y)$ top views, $z=40 \mathrm{~nm}$ (center of the nanoparticles). (j-l) Cross sections and top views of the simulated near field (TE) with one harmonic at the selected wavelengths. Black dashed lines indicate the interfaces. TM near fields are provided in Figure S6b, Supporting information 


\subsection{Conditions for multiplexing and color search algorithm}

In image multiplexing, different images are combined to form a multiplexed image whose bit depth is the sum of the bit depths of each initial image. In the two examples illustrated in Figure 5, Figure $5 a$ and $5 d$ show the images to be produced, and Figure $5 \mathrm{~b}$ and $5 \mathrm{e}$ the respective multiplexed images. Implementing printed image multiplexing requires finding out, among the full set of metasurfaces produced by laser processing, specific sub-sets that form a logical color tree in selected observation modes, as illustrated in Figure $5 \mathrm{c}$ and $5 f$. When selecting $M$ modes and $K$ colors per mode, a logical color tree gathers $K^{M}$ types of metasurfaces that provide all the possible combinations of the $K$ colors in the $M$ modes. The examples in Figure 5 illustrate the cases where $K=2$ and $M=2$ or $K=2$ and $M=3$ that are used to display multiplexed bicolor images in either 2 or 3 modes, respectively. In such cases, 4 (for $M=2$ ) or 8 (for $M=3$ ) different metasurfaces exhibiting the specific combinations of colors shown in Figure $5 c$ and $5 \mathrm{f}$ must be found in the set of laser-processed metasurfaces.

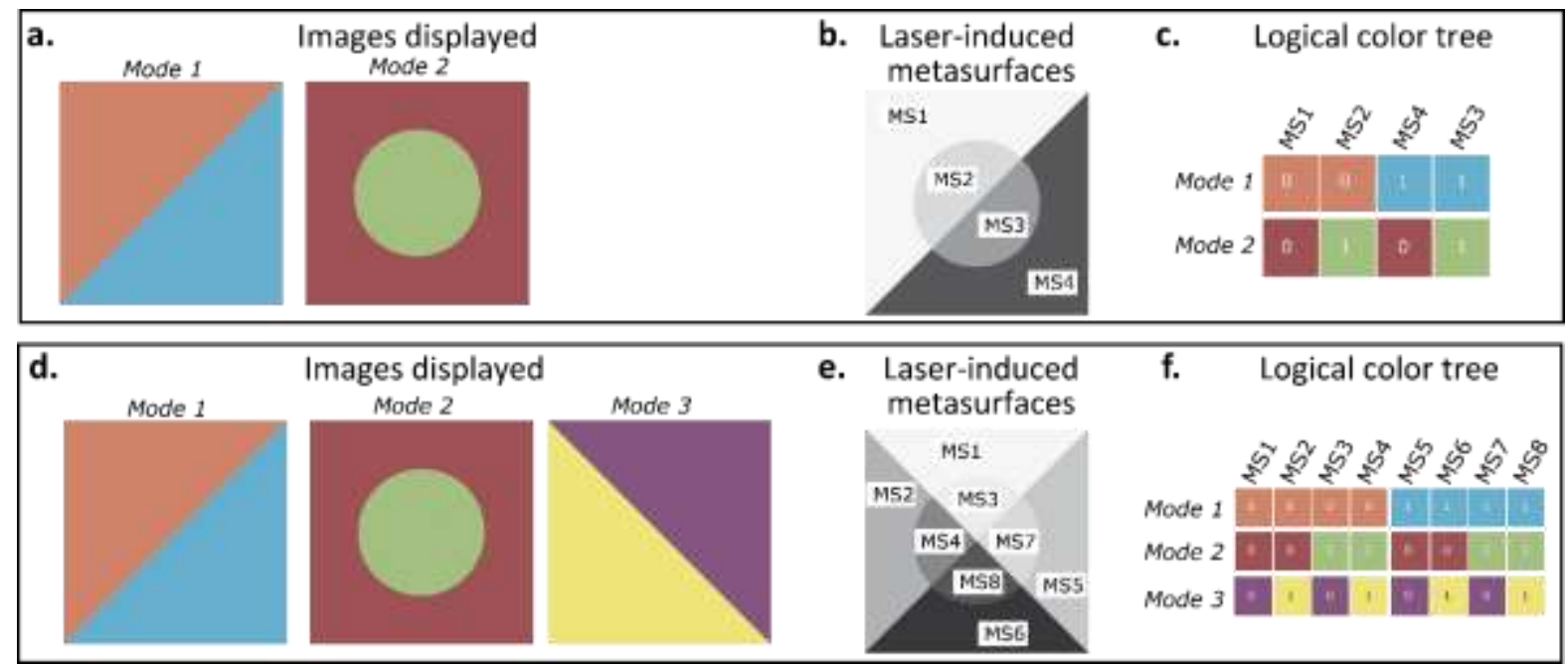

Figure 5. Principle of image multiplexing for 2 and 3 modes (a) 2 bicolor images to be displayed in mode 1 and 2. (b) 4 laser-induced metasurfaces (MS) are needed to multiplex the two images. (c) The colors of the 4 metasurfaces in modes 1 and 2 form a logical color tree with 2 modes and 2 colors per mode. (d) 3 bicolor images to be displayed in mode 1, 2 and 3. (e) 8 distinct metasurfaces are needed to multiplex the three images. (f) The colors in modes 1, 2 and 3 of the 8 metasurfaces form a logical color tree with 3 modes and 2 colors per mode.

The following search algorithm is proposed to find all the possible solutions that enable multiplexing within a data set such as the one presented in Figure S3, Supporting Information, which includes 9072 metasurfaces providing a large range of colors measured in various modes:

1. The colors of $N$ laser-processed metasurfaces are measured in the selected modes. Colors can be expressed in any color space in which there exists a relevant colorimetric distance in respect to the observation conditions. Here, the CIE1976 $\mathrm{L}^{*} \mathrm{a}^{*} \mathrm{~b}^{*}$ color space is used (see Supporting
Note 3 for more details on the color conversion), with the $\Delta L^{*}$ perceptual distance. 2. In each mode, a maximum distance hierarchical clustering is performed by using the chosen perceptual color distance to form clusters of nanostructured pixels having a distance smaller than an arbitrary cut-off distance in each mode. Each nanostructured pixel belongs to one and only one cluster in each mode. The total number of clusters in each mode can vary. Let $C_{i}^{m}$ be the cluster number $i$ in mode $m$.

3. For each pair if $M=2$ (respectively triplet, if $M=3$ ) of selected modes: 
3.1. For each pair of clusters in mode $1, C_{i}^{1}$ and $C_{j}^{1}$, which contain at least two (respectively four) metasurfaces each, identify all the clusters $C_{k}^{2}$ in mode 2 that contain at least one (respectively two) metasurface(s) belonging to $C_{i}^{1}$ and one (respectively two) metasurface(s) belonging to $C_{j}^{1}$.

3.1.1. Case where $M=2$ : If there are at least two clusters $C_{k}^{2}$ and $C_{l}^{2}$ that satisfy the condition 3.1, then any combination of 4 metasurfaces that belong respectively to the four sets: $S_{1}=C_{i}^{1} \cap C_{k}^{2}, S_{2}=C_{i}^{1} \cap C_{l}^{2}, S_{3}=$ $C_{j}^{1} \cap C_{k}^{2}$ and $S_{4}=C_{j}^{1} \cap C_{l}^{2}$ is a solution for two-image multiplexing with two colors per mode.

3.1.2. Case where $M=3$ : If there are at least two clusters $C_{k}^{2}$ and $C_{l}^{2}$ that satisfy the condition 3.1 with at least two metasurfaces per cluster intersection, identify all the clusters $C_{m}^{3}$ in mode 3 that contain at least one metasurface belonging to each cluster intersection $S_{1}, S_{2}, S_{3}$ and $S_{4}$. If there are at least two clusters $C_{m}^{3}$ and $C_{n}^{3}$ that satisfy this condition, then any combination of 8 metasurfaces that belongs respectively to the eight sets: $R_{1}=S_{1} \cap C_{m}^{3}, R_{2}=S_{1} \cap C_{n}^{3}, R_{3}=$ $S_{2} \cap C_{m}^{3}, R_{4}=S_{2} \cap C_{n}^{3}, R_{5}=S_{3} \cap C_{m}^{3}, R_{6}=$ $S_{3} \cap C_{n}^{3}, R_{7}=S_{4} \cap C_{m}^{3}$ and $R_{8}=S_{4} \cap C_{n}^{3}$ is a solution for three-image multiplexing with two colors per mode.

This conditional architecture can extend to more than three modes and also to more than two colors per mode. In order to propose the best image contrast for the two (respectively three) multiplexed images, an additional step filters the solutions to keep only those that give both the highest colorimetric distance and the highest contrast (lightness difference) between the two selected colors in each mode. The user can then select among the proposed solutions those whose colors better fit his expectations.

The algorithm was implemented on the sets of RGB colors measured in seven different modes shown in Figure S3, Supporting Information. This approach is adapted to deal with the laserinduced random plasmonic metasurfaces, whose optical properties are difficult to predict. This algorithm can also apply to other printing technologies and could suggest nonobvious color combinations, which increase the inherent security of these techniques. The following sections demonstrate printed image multiplexing with 2, 3 or 4 images, different mode combinations and various color numbers per mode; highlighting the potential of combining $\mathrm{Ag}: \mathrm{TiO}_{2}$ thin films and laserprocessing for security printing.

\subsection{Two- and three-image multiplexing with naked eye observation}

This section focuses on modes that can be observed by naked eye to demonstrate the possibility to produce overt security features with two- or three-image multiplexing. Figure 6 gathers the true color images, recorded under white light with the setup depicted in Figure S7, Supporting Information, of four nanosecondlaser-processed samples for which multiplexing is implemented for different combinations of modes. To limit the number of different metasurfaces in each multiplexed image, the number of colors per mode is limited to two. Gradients in images are rendered using a dithering algorithm described in Supporting Note 2, as well as the procedure used to create the encoded images, which contain the information of the two or three multiplexed images. All the laser processing parameter sets used to print the multiplexed images of Figure 6 are given in Table S3, Supporting Information. The first demonstration involves "transmission" and "diffraction" modes with pictures of a butterfly and a raccoon (Figure $6 a)$. The four selected metasurfaces contain self-organized gratings whose orientation is parallel to the incident laser polarization. They are produced with two laser fluences, which give rise to two different colors in transmission and similar colors in the diffraction mode. Each couple (MS1, MS2) or (MS3, MS4) is produced with the same laser fluence. The laser polarization is chosen to create gratings that diffract in the observation plane for MS2 and $\mathrm{MS3}$, and is rotated by $60^{\circ}$ to diffract out of the observation plane for MS1 and MS4 as shown in the color tree below the photographs. The true size of the printed images is $13.4 \times 15.9$ $\mathrm{mm}^{2}$ with a pixel size of $72 \mu \mathrm{m}$. Supporting Movie 1 shows the dynamic reading of the multiplexed images with a smartphone camera moving from one mode to the other. 
The second demonstration involves modes "backside reflection" and "transmission" with portraits of two of the authors (Figure 6b). The image size is $5.4 \times 8.0 \mathrm{~mm}^{2}$ with a pixel size of $20 \mu \mathrm{m}$. The four metasurfaces selected for implementing multiplexing involve parameter sets with various laser fluences, repetition rates and pulse numbers. Their colors in the two modes are arranged in the color tree. Supporting Movie 2 shows a dynamic reading of the multiplexed images with a smartphone camera that switches from the "backside reflection" mode to the "transmission" mode.

The third sample demonstrates three-image multiplexing observable with naked eye under white light. It involves modes "scattering", "transmission" and "diffraction" with photographs of three PhD students of our team (Figure 6c). The image size is $10.9 \times 15.8 \mathrm{~mm}^{2}$ with a pixel size of $72 \mu \mathrm{m}$. The eight metasurfaces selected for implementing multiplexing involve parameter sets with various laser fluences, speeds, repetition rates, polarizations and interline spacing values. Their colors in the three modes are arranged in the color tree. Supporting Movie 3 shows a dynamic reading of the three multiplexed images with a smartphone camera.
In Figure 6d, seven "primary" colors are selected in the "backside reflection" mode to print a color image. As detailed in Supporting Note 3 , halftoning of these colors enables rendering the other tones. The colors of the initial image are converted to the experimental ones by gamut mapping and quantization, further a dithering algorithm is used for rendering color gradients. The second mode here is the "diffraction" one. Among the seven selected metasurfaces, only one diffracts light (MS7). The latter is duplicated with a different grating direction in MS8 giving the same color in "backside reflection" and a different color in "diffraction". Logos are thus inserted in the "backside reflection" colored image in the areas where the MS7/MS8 color is present. In this example, the repetition rate and the interline spacing are fixed, while the fluence, polarization and scanning speed are varied. Further, some colors are produced by repeating the laser processing several times in the same pixel introducing another parameter shown in the Table S3, Supporting Information (number of passes). Supporting Movie 4 shows a dynamic reading of the color image and the diffracting logos with a smartphone camera. 

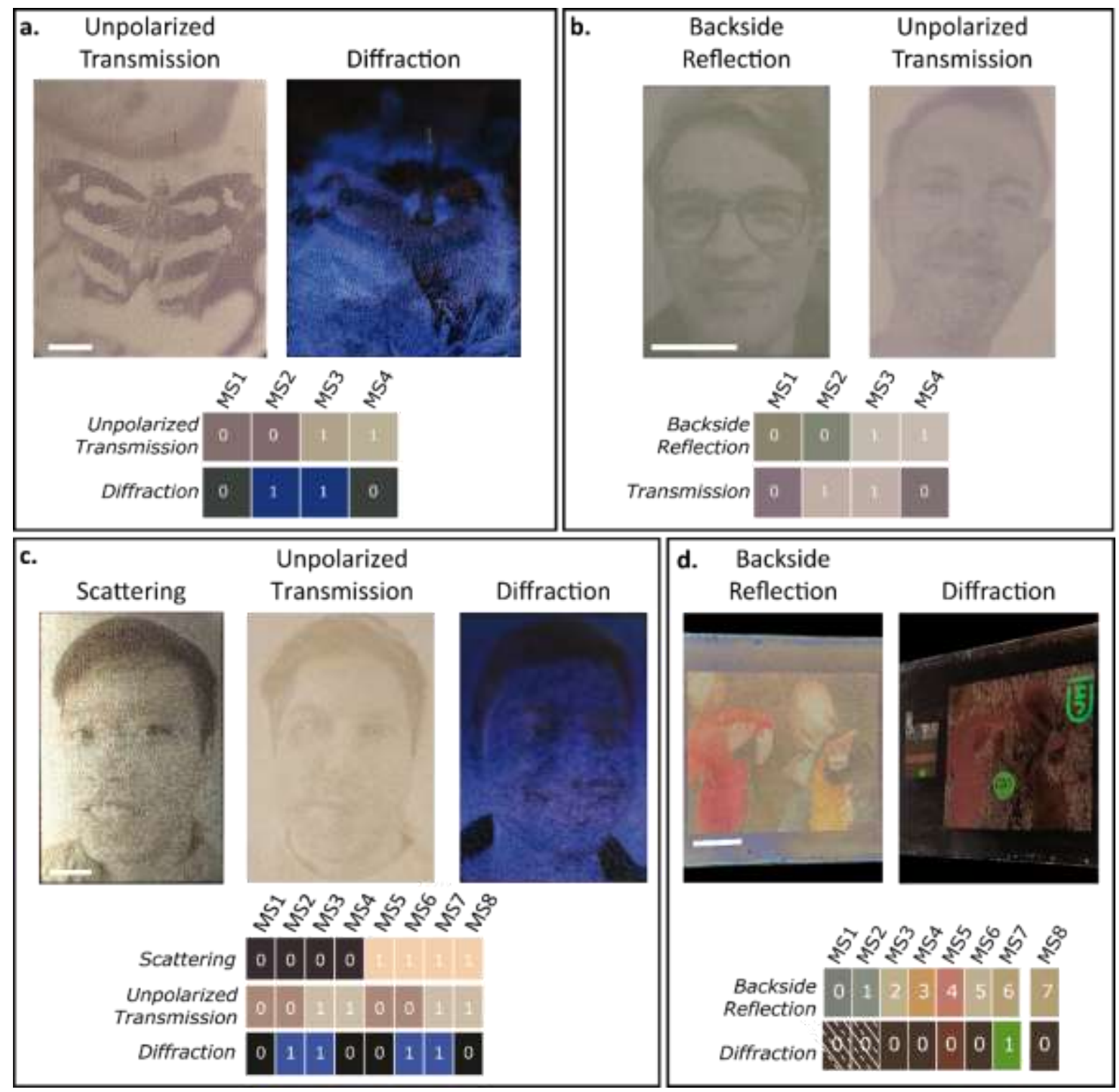

Figure 6 Image multiplexing with naked eye observation under unpolarized white light. Images recorded in different modes and logical color trees showing the colors of the used metasurfaces in the different modes. (a) Two-image multiplexing with two colors per mode observed in modes "transmission" and "diffraction". (b) Two-image multiplexing with two colors per mode observed in modes "backside reflection" and "transmission". c. Three-image multiplexing with two colors per mode observed in modes "scattering", "transmission" and "diffraction". (d) Twoimage multiplexing with 7 and 2 colors in mode "backside reflection" and "diffraction", respectively. Original image of the two macaws reproduced with permission from the Kodak Photo CD Sampler. ${ }^{[51]}$ The length of the scale bars is $2.5 \mathrm{~mm}$ in all images.

\subsection{Four-image multiplexing using polarized modes}

White light three-image multiplexing has been recently demonstrated using femtosecond laser processing with images displayed in the following modes: reflection with non-polarized light and transmission with the sample placed between two polarizers ${ }^{[37]}$. It was also demonstrated that with an anisotropic sample,

two images can always be multiplexed when using polarized light provided that two azimuthal angles $\alpha$ of the sample are used to display two images ${ }^{[37]}$. Here, the methodology is similar as illustrated previously, but the three images are observed in transmission only. One is revealed under unpolarized light, the two 
other ones being revealed when the sample is placed between two polarizers with two different rotation angles of the sample in its plane. Furthermore, a blank image, hiding the three images, appears in "frontside reflection" mode.

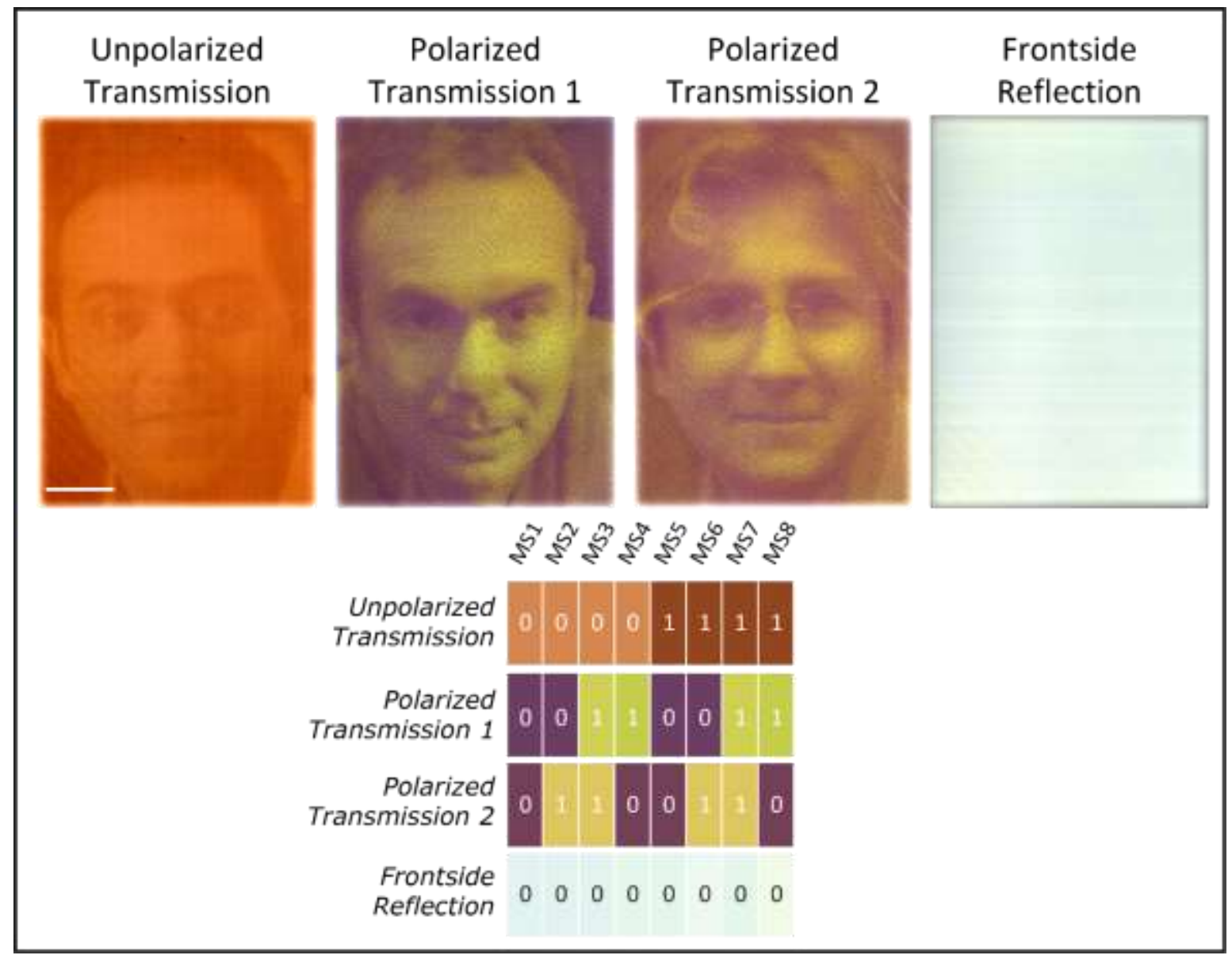

Figure 7. Four-image multiplexing with polarized white light. Three images and a blank image are multiplexed to be revealed in "unpolarized transmission", "polarized transmission 1", "polarized transmission 1" and "frontside reflection". The pictures are the photographs of the sample taken with our setup in the corresponding modes. The colors of the eight metasurfaces are displayed in the logical color tree. The length of the scale bar is $2.5 \mathrm{~mm}$.

Apart the blank image, bicolor images are used for this four-image multiplexing demonstration, and dithering is used to render color gradients in each mode. The eight metasurfaces selected for this implementation all exhibit dichroism caused by the generation of self-organized nanoparticle gratings parallel to the laser polarization. Two speeds (50 and $200 \mathrm{~mm} \mathrm{~s}^{-1}$ ) are used to get two different colors in the non-polarized transmission mode. Four laser polarization orientations $\left(30^{\circ}, 60^{\circ}, 120^{\circ}\right.$ and $150^{\circ}$ ) are used to get two color combinations between the two polarizers (at $\beta$ $=85^{\circ}$ from each other) for two specific azimuthal angles $\alpha$ of the sample. The colors of these eight metasurfaces in four modes are shown in the logical color tree in Figure 7, together with the photographs of the pictures revealed in each mode. They show three of the authors. The first one appears in the nonpolarized transmission mode, whereas the two others appear in the polarized transmission modes. Supporting Movie 5 shows the morphing that appears in the polarized transmission mode when rotating the sample in its plane (azimuthal angle $\alpha$ ) between the two specific angles where each image alternatively appears. Table S4, Supporting Information gives all the processing parameters for each metasurface.

This demonstration of four-image multiplexing proves that various solutions can be found for 
multiplexing provided that the general methodology described in this paper is implemented to find them out. The speeds used with this nanosecond laser processing are in average about 50 times higher than the ones reported with the femtosecond laser processing and allowed to produce the presented demonstrators in 5 to 10 min each. Both the lower cost of the laser and high speed of the process make the industrial implementation feasible.

\section{Conclusion}

The paper presents an overview of the high variety of random plasmonic metasurfaces that can be produced by nanosecond laser processing of $\mathrm{Ag}: \mathrm{TiO}_{2}$ thin films, and show examples of the large number of color combinations that are provided by these metasurfaces in the seven observation modes that have been selected for the demonstration. The structure of random plasmonic metasurfaces is statistically controlled by the laser process, thus making their prediction difficult. It is also complex to think of predicting their optical properties with an optical model in order to simulate their color in different observations scenarios, which is an advantage for security applications. Electromagnetic modeling is carried out on a simplified structure to evidence the role of hybridization between lattice resonances and localized surface plasmon resonances on the optical dichroism of these metasurfaces. To overcome the lack of predictive model between the laser processing parameters and the resulting colors, we propose a method relying on the color measurement of a set of random plasmonic metasurfaces in the selected modes, and on an algorithm allowing the identification of the metasurfaces enabling printed image multiplexing. Two- and three-image multiplexing are demonstrated for the first time with observation modes that do not need any particular tool to reveal the images independently, producing thus overt security features. Naked eye visualization under incoherent white light allows observing different images in different combinations of modes among which "transmission", "backside reflection", "frontside reflection", "scattering" and "diffraction" configurations. Even though the use of polarizers is not possible in overt security applications, polarization modes can be added to the previous set of modes, which demonstrate the versatility of this technology and its ability to extend to covert security applications. A four-image multiplexing demonstrator has been produced, by displaying three different images and one blank image. Halftoning is used to print grayscale and full color images with a limited encoding complexity of multiplexed images and up to six laser-processing parameters are controlled to reach these outcomes. The use of a nanosecond laser allows reaching processing speeds that are about fifty times higher than the best ones previously reported with femtosecond lasers for the same kind of applications, and also demonstrates higher flexibility with a larger variety of modes. This work opens the way to the industrial implementation of image multiplexing in security printing for smartphone-basedreading or for naked eye control, with the ability to print customized overt security features with a low-cost nanosecond laser.

\section{Experimental Section/Methods}

Ag: TiO $_{2}$ initial fim: Microscopy glass slides are coated with a mesoporous thin film of amorphous $\mathrm{TiO}_{2}$ impregnated with silver salt. The mesoporous titania film is elaborated by a sol-gel process (detailed in Method S1, Supporting Information) forming mesoporous titania films with a thickness of $200 \pm 30 \mathrm{~nm}$. The titania pores are then filled with silver ionic solution, by soaking the films in $0.85 \mathrm{M}$ silver ionic solution. Ions are converted to atoms and nanoparticles, whose size is in the 1-3 nm range, by UV irradiation $\left(254 \mathrm{~nm}, 6 \mathrm{~mW} \mathrm{~cm}^{-2}, 5\right.$ $\mathrm{min}$ ). All this process has already been used in references. ${ }^{[37,53]}$

Nanosecond laser processing: An industrial fibered laser (IPG Photonics) of wavelength 532 $\mathrm{nm}$ with a typical pulse duration of $1.3 \mathrm{~ns}$ is scanned over the sample with a galvanometric mirror scanner head (Sunny Technology). The laser is linearly polarized using a Brewster angle polarizer placed before the scanner head (Figure S1, Supporting Information). The polarization can be rotated using a half-wave plate. The laser beam is focused on the sample 
using a $16 \mathrm{~cm}$ F-Theta lens providing a focused laser spot size of $13.5 \mu \mathrm{m}$ with a Rayleigh length of $818 \mu \mathrm{m}$ over a maximum field size of $11 \times 11 \mathrm{~cm}^{2}$ in the focal plane. Images are printed with two types of pixels (Figure S2, Supporting Information). The largest one is a $72^{2} \mu m^{2}$ square pixel printed by filling the square area with overlapping laser lines parallel to the pixel diagonal and separated by an interline spacing $d y$, which fixes the overlapping rate. The smallest pixel is filled with a static laser spot printed with various fluences, pulse numbers, polarizations and repetition rates; it has a size of $20^{2} \mu \mathrm{m}^{2}$ fixed by the distance set between the spots center.

Optical Characterizations: A telecentric lens images the sample surface illuminated by a 15 $\mathrm{cm}$ wide LED light source on a RGB camera (Basler ac2500-14gc). Measurements were carried out with a mean incidence angle of $0^{\circ}$ in transmission and of $16^{\circ}$ in reflection. In the scattering mode, the camera axis is normal to the sample surface and the incidence angle is $32^{\circ}$. Diffraction catches the $-1^{\text {st }}$ diffraction order for an incidence angle of $64^{\circ}$. For the polarized transmission modes, one polarizer is placed between the source and the sample, and another one between the sample and the camera. The polarizers can be rotated independently in their plane. In the sketches of the polarized modes $f$ and $g$, the azimuthal angle $\alpha$ of the sample corresponds to the angle of the horizontal side of a square relative to the polarizer axis. It is set to $45^{\circ}$ in $\mathrm{f}$ and to $135^{\circ}$ in g. Angle $\beta$ corresponds to the angle of the analyzer axis relative to the polarizer axis, which is set to $85^{\circ}$ in $\mathrm{f}$ and $\mathrm{g}$. When available, the spectra were recorded by replacing the light source by a supercontinuum laser (Newwave, Leukos) with a wavelength-tunable filter (BEBOP, Leukos), acquiring hyperspectral images of each square normalized to the unprocessed film and multiplied by the transmittance or reflectance of the film to recover the spectra. Gamuts were obtained by extracting the RGB color from the color images of the complete database, converting to $\mathrm{CIE}$ L*a*b coordinates (see Supplementary Note 3 ) and projecting onto the $a^{*} b^{*}$ plane (colored dots). Chromaticity diagrams in the CIE 1931 xy space computed from the intermediate $X Y Z$ tristimulus values are provided in Figure S4, Supporting information.

Other characterizations: A FEI Nova nanOSEM 200 scanning electron microscope (SEM) is used in low vacuum mode with a helix detector to measure the surface morphology. This allows to measure the morphology of the sample under vacuum without any conductive coating. A Bruker Icon atomic force microscope (AFM) is used in tapping mode to measure the surface topography. Lamellae for TEM observation are prepared with a FIB-FEI Helios $600 \mathrm{i}$ instrument and measured with a Jeol 2010 TEM operated at $200 \mathrm{kV}$.

Electromagnetic modeling: All simulations are carried out with a homemade code that allows for solving rigorously the scattering problem in a multilayer. The theoretical approach is the same as the one of the free access code Smuthi. ${ }^{[54]}$ It involves transition matrices to calculate multiple scattering by the nanoparticles $^{[55]}$ and a scattering matrix formalism to account for the multiple reflections on plane interfaces. ${ }^{[56]}$ Once the scattering problem is solved, the near field can be calculated everywhere except inside the spherical nanoparticles, and the total field can be expanded in terms of outgoing spherical vector wave functions or plane vector wave functions at any position $\boldsymbol{r} \cdot{ }^{[54]}$ In presence of plane interfaces, the extinction cross section is the sum of two terms, the powers taken from the reflected and transmitted waves divided by the intensity of the incoming exciting plane wave incident on the interface. Each extinction term can be expressed using the generalized optical theorem ${ }^{[57-60]}$ and results from the interference between the specularly reflected $\left(E_{i n c, R}^{*}\right)$ or transmitted $\left(E_{i n c, T}^{*}\right)$ field (without any particle in the system) with the far field scattered in the same directions, $E_{s c a, R}^{\infty}\left(\boldsymbol{k}_{R}\right)$ or $E_{s c a, T}^{\infty}\left(\boldsymbol{k}_{T}\right)$, respectively:

$$
\begin{aligned}
& C_{\text {ext }, R}=\frac{4 \pi}{k_{R}\left|E_{i n c, R}\right|^{2}} \operatorname{Im}\left(E_{i n c, R}^{*}\right. \\
& C_{\text {ext }, T}=\frac{4 \pi}{k_{T}\left|E_{\text {inc }, T}\right|^{2}} \operatorname{Im}\left(E_{\text {inc, } T}^{*}\right. \\
& \left.\cdot E_{s c a, T}^{\infty}\left(\boldsymbol{k}_{T}\right)\right)
\end{aligned}
$$


$\boldsymbol{k}_{R}$ and $\boldsymbol{k}_{T}$ are the wavevectors of the reflected and transmitted waves in the air and the glass, respectively. $E^{*}$ stands for the complex conjugate of the field $E$. Although $C_{\text {ext }}$ must be positive, $C_{\text {ext }, R}$ and $C_{\text {ext }, T}$ can be negative since the nanoparticle scattering can increase the power in the specular directions compared to what the multilayer provides without the nanoparticles.

An estimate of the transmission ( $T$ ) and reflection ( $R$ ) coefficients can be deduced from the following equations:

$$
\begin{gathered}
T=\frac{P_{T}}{P_{i}}=\frac{P_{T, \text { layer }}-P_{N P}+P_{S c a}(\Omega)}{P_{i}} \\
=\frac{\Delta S I_{T, \text { layer }}-C_{\text {ext }, T} I_{T, \text { layer }}+P_{\text {sca }}(\Omega)}{\Delta S I_{i}} \\
\approx \frac{I_{T, \text { layer }}}{I_{i}}-\frac{I_{T, \text { layer }}}{I_{i}} \frac{C_{\text {ext }, T}}{\Delta S}
\end{gathered}
$$

Where $P_{i}$ and $P_{T}$ are the incident and transmitted power, $P_{T, \text { layer }}$ is the power transmitted by the layer system only, $P_{N P}$ is the extinguished power from NPs and $P_{s c a}(\Omega)$ is the power scattered within the solid angle $(\Omega)$ of a detector of size $\Delta S$. When neglecting the power scattered in the solid angle of the detector relative to the total extinguished power, one can approximate the transmission coefficient by:

$$
T \approx \frac{I_{T}}{I_{i}}\left(1-\frac{C_{\text {ext }, T}}{\Delta S}\right)
$$

And similarly, the reflection coefficient can be approximated by:

$$
R \approx \frac{I_{R}}{I_{i}}\left(1-\frac{C_{e x t, R}}{\Delta S}\right)
$$

The simulations are carried out using a modified Drude model to fit the metal dielectric permittivity of silver (the used Drude parameters are the same as in $\operatorname{Re}^{[59]}$ ), by considering 3 harmonics for the calculation of the scattered field and choosing $\Delta S=$ $\max _{\lambda}\left(\left|C_{\text {ext }, T}\right|+\left|C_{\text {ext }, R}\right|\right)$

\section{Supporting Information}

Supporting Information is available from the Wiley Online Library or from the author.

\section{Acknowledgements}

This work was funded by HID Global CID and the ANR project MIXUP (ANR-18-CE39-0010). The authors thank David Troadec for the preparation of the FIB lamellae and Lucian Roiban for the TEM measurements.

\section{References}

[1] C. Ng, L. Wesemann, E. Panchenko, J. Song, T. J. Davis, A. Roberts, D. E. Gómez, Adv. Opt. Mater. 2019, 7, 1801660.

[2] V. J. Cadarso, S. Chosson, K. Sidler, R. D. Hersch, J. Brugger, Light Sci. Appl. 2013, 2, e86.

[3] J. Andres, R. D. Hersch, J.-E. Moser, A.-S. Chauvin, Adv. Funct. Mater. 2014, 24, 5029.

[4] C. Zhang, B. Wang, W. Li, S. Huang, L. Kong, Z. Li, L. Li, Nat. Commun. 2017, 8, 1138.

[5] C. Fuller, A. Chen, Meas. Sci. Technol. 2011, 22, 105703.

[6] G. Howells, R. J. Prance, T. D. Clark, H. Prance, Meas. Sci. Technol. 1997, 8, 734 .

[7] H. I. Bjelkhagen, Opt. Eng. 1999, 38, 55.

[8] T. Walger, T. Besson, V. Flauraud, R. D. Hersch, J. Brugger, Opt. Express 2019, 27, 37419.

[9] A. Goncharsky, A. Goncharsky, D. Melnik, S. Durlevich, Sci. Rep. 2021, 11, 2426.

[10] W. Ren, G. Lin, C. Clarke, J. Zhou, D. Jin, Adv. Mater. 2020, 32, 1901430.

[11] P. Pjanic, R. D. Hersch, ACM Trans. Graph. 2015, 34, 130:1.

[12] N. Dalloz, M. Hébert, in Comput. Color Imaging (Eds.: S. Tominaga, R. Schettini, A. Trémeau, T. Horiuchi), Springer International Publishing, 2019, pp. 63-76.

[13] J. Scheuer, ACS Photonics 2020, 7, 1323.

[14] X. Zhu, C. Vannahme, E. HøjlundNielsen, N. A. Mortensen, A. Kristensen, Nat. Nanotechnol. 2016, 11,325 . 
[15] M. Keshavarz Hedayati, M. Elbahri, Plasmonics 2017, 12, 1463.

[16] Y.-W. Huang, W. T. Chen, W.-Y. Tsai, P. C. Wu, C.-M. Wang, G. Sun, D. P. Tsai, Nano Lett. 2015, 15, 3122.

[17] W. Wan, J. Gao, X. Yang, ACS Nano 2016, 10, 10671.

[18] U. Levy, E. Marom, D. Mendlovic, Opt. Lett. 2001, 26, 1149.

[19] B. Wang, F. Dong, Q.-T. Li, D. Yang, C. Sun, J. Chen, Z. Song, L. Xu, W. Chu, Y.-F. Xiao, Q. Gong, Y. Li, Nano Lett. 2016, 16, 5235.

[20] W. Ye, F. Zeuner, X. Li, B. Reineke, S. He, C.-W. Qiu, J. Liu, Y. Wang, S. Zhang, T. Zentgraf, Nat. Commun. 2016, 7, 11930.

[21] W. T. Chen, K.-Y. Yang, C.-M. Wang, Y.-W. Huang, G. Sun, I.-D. Chiang, C. Y. Liao, W.-L. Hsu, H. T. Lin, S. Sun, L. Zhou, A. Q. Liu, D. P. Tsai, Nano Lett. 2014, 14, 225.

[22] J. P. Balthasar Mueller, N. A. Rubin, R. C. Devlin, B. Groever, F. Capasso, Phys. Rev. Lett. 2017, 118, 113901.

[23] L. Jin, Z. Dong, S. Mei, Y. F. Yu, Z. Wei, Z. Pan, S. D. Rezaei, X. Li, A. I. Kuznetsov, Y. S. Kivshar, J. K. W. Yang, C.-W. Qiu, Nano Lett. 2018, 18, 8016.

[24] Y. Montelongo, J. O. Tenorio-Pearl, W. I. Milne, T. D. Wilkinson, Nano Lett. 2014, 14, 294.

[25] B. Wang, F. Dong, D. Yang, Z. Song, L. Xu, W. Chu, Q. Gong, Y. Li, Optica 2017, 4, 1368.

[26] S. M. Kamali, E. Arbabi, A. Arbabi, Y. Horie, M. Faraji-Dana, A. Faraon, Phys. Rev. X 2017, 7, 041056.

[27] H. Ren, X. Li, Q. Zhang, M. Gu, Science 2016, 352, 805.

[28] Y. Hu, X. Luo, Y. Chen, Q. Liu, X. Li, Y. Wang, N. Liu, H. Duan, Light Sci. Appl. 2019, 8 .

[29] K. T. P. Lim, H. Liu, Y. Liu, J. K. W. Yang, Nat. Commun. 2019, 10.

[30] P. Zijlstra, J. W. M. Chon, M. Gu, Nature 2009, 459, 410.

[31] Y. Bao, Y. Yu, H. Xu, Q. Lin, Y. Wang, J. Li, Z.-K. Zhou, X.-H. Wang, Adv. Funct. Mater. 2018, 28, 1805306.
[32] M. Song, Z. A. Kudyshev, H. Yu, A. Boltasseva, V. M. Shalaev, A. V. Kildishev, Opt. Mater. Express 2019, 9, 779 .

[33] L. Wang, T. Li, R. Y. Guo, W. Xia, X. G. Xu, S. N. Zhu, Sci. Rep. 2013, 3.

[34] H. Liu, J. Xu, H. Wang, Y. Liu, Q. Ruan, Y. Wu, X. Liu, J. K. W. Yang, Adv. Mater. 2019, 31, 1807900.

[35] X. M. Goh, Y. Zheng, S. J. Tan, L. Zhang, K. Kumar, C.-W. Qiu, J. K. W. Yang, Nat. Commun. 2014, 5, 5361.

[36] E. Heydari, J. R. Sperling, S. L. Neale, A. W. Clark, Adv. Funct. Mater. 2017, 27, 1701866.

[37] N. Destouches, N. Sharma, M. Vangheluwe, N. Dalloz, F. Vocanson, M. Bugnet, M. Hébert, J. Siegel, $A d v$. Funct. Mater. 2021, 2010430.

[38] Q. Dai, N. Zhou, L. Deng, J. Deng, Z. Li, G. Zheng, Phys. Rev. Appl. 2020, 13, 034002.

[39] W. Dorfler, A. Assfalg, Identification Document Having a Personalized Visual Identifier and Method for Production Thereof, 2015, US8998264B2.

[40] N. Sharma, N. Destouches, C. Florian, R. Serna, J. Siegel, Nanoscale 2019, $11,18779$.

[41] Z. Liu, J. Siegel, M. Garcia-Lechuga, T. Epicier, Y. Lefkir, S. Reynaud, M. Bugnet, F. Vocanson, J. Solis, G. Vitrant, N. Destouches, ACS Nano 2017, 11, 5031.

[42] N. Destouches, N. Crespo-Monteiro, G. Vitrant, Y. Lefkir, S. Reynaud, T. Epicier, Y. Liu, F. Vocanson, F. Pigeon, J Mater Chem $C$ 2014, 2, 6256.

[43] A. Podlipensky, A. Abdolvand, G. Seifert, H. Graener, Appl. Phys. A 2005, 80, 1647.

[44] M. Dupré, L. Hsu, B. Kanté, Sci. Rep. 2018, 8 .

[45] N. Sharma, M. Vangheluwe, F. Vocanson, A. Cazier, M. Bugnet, S. Reynaud, A. Vermeulin, N. Destouches, Mater. Horiz. 2019, 6, 978. 
[46] Z. Liu, G. Vitrant, Y. Lefkir, S. Bakhti, N. Destouches, Phys. Chem. Chem. Phys. 2016, 18, 24600.

[47] S. Zou, N. Janel, G. C. Schatz, J. Chem. Phys. 2004, 120, 10871.

[48] M. B. Ross, C. A. Mirkin, G. C. Schatz, J. Phys. Chem. C 2016, 120, 816.

[49] A. D. Utyushev, V. I. Zakomirnyi, I. L. Rasskazov, Rev. Phys. 2021, 6, 100051.

[50] V. Giannini, Y. Francescato, H. Amrania, C. C. Phillips, S. A. Maier, Nano Lett. 2011, 11, 2835.

[51] D. Khlopin, F. Laux, W. P. Wardley, J. Martin, G. A. Wurtz, J. Plain, N. Bonod, A. V. Zayats, W. Dickson, D. Gérard, J. Opt. Soc. Am. B 2017, 34, 691.

[52] R. Franzen, "True Color Kodak Images," can be found under http://r0k.us/graphics/kodak/, 2021.

[53] F. Tricot, F. Vocanson, D. Chaussy, D. Beneventi, S. Reynaud, N. Destouches, RSC Adv. 2014, 9.

[54] A. Egel, Accurate optical simulation of disordered scattering layers for light extraction from organic light emitting diodes, Karlsruher Institut für Technologie, 2019.

[55] D. W. Mackowski, J. Quant. Spectrosc. Radiat. Transf. 2008, 109, 770.

[56] A. Egel, U. Lemmer, J. Quant. Spectrosc. Radiat. Transf. 2014, 148, 165.

[57] D. Torrungrueng, B. Ungan, J. T. Johnson, IEEE Geosci. Remote Sens. Lett. 2004, 1, 131.

[58] D. R. Lytle, P. S. Carney, J. C. Schotland, E. Wolf, Phys. Rev. E 2005, 71, 056610.

[59] N. Dahan, J.-J. Greffet, Opt. Express 2012, 20, A530.

[60] M. Pellarin, C. Bonnet, J. Lermé, F. Perrier, J. Laverdant, M.-A. Lebeault, S. Hermelin, M. Hillenkamp, M. Broyer, E. Cottancin, J. Phys. Chem. C 2019, 123, 15217.

[59] S. Bakhti, N. Destouches, A. V. Tishchenko, J. Quant. Spectrosc. Radiat. Transfer 2014, 146:113. 